\title{
A CLASS OF SELF-SIMILAR NETWORKS
}

\author{
Vesna Andova, Pavel Dimovski \\ Faculty of Electrical Engineering and Information Technologies, \\ "Ss. Cyril and Methodius" University in Skopje, \\ Rugjer Bošković bb, P.O. box 574, 1001 Skopje, North Macedonia \\ vesnaa@feit.ukim.edu.mk
}

\begin{abstract}
A b s t r a c t: Large graphs, also known as networks, are often used to represent different natural or human made structures. In many of these networks the phenomenon of self-similarity was detected. Here we present a deterministic method for construction of a class of self-similar networks. The elements of this class are obtained by a vertex-transitive graphs, and an iterative construction procedure implemented on these graphs. This procedure provides the property of self-similarity in the obtained network.
\end{abstract}

Key words: graph; networks; self-similarity

\section{КЛАСА САМОСЛИЧНИ МРЕЖИ}

А п с т р а к т: Големите графови (или мрежи) често се користат за визуелно претставување на различни природни мрежи или мрежи креирани од човекот. Кај многу од овие мрежи се забележува феноменот на самосличност. Во овој труд е даден детерминистички метод за конструкција на класа самослични мрежи. Елементите од оваа класа се добиени од транзитивни графови со примена на итеративна конструктивна постапка. Овој конструктивен метод го обезбедува својството на самосличност на добиената мрежа.

Клучни зборови: графови; мрежи; самосличност

\section{INTRODUCTION}

A network (or graph) is simply a collection of connected objects (by some property). That is why the saying "A picture says more than a thousand words" is the best descriptions why graphs, also known as networks, are one of the most common model in modern science for describing deferent natural or a human-made structures. Using graphs as a visual representation of the data one can gain actionable insights and make better data driven decisions.

The interest in studying networks correlated with complex system like society, world wide web, statistical physics, particle physics, computer science, electrical engineering [1,2], biology, economics, finance etc., was mainly motivated by the publication of Watts and Strogatz [3] on small-world networks, and by Baràbasi and Albert [4] on scalefree networks.

This approach gives an abstract perspective to the problem: electrical networks can be considered regardless of the electric power; social networks can be considered regardless to religion, political orientation, etc. At the same time weights can be assigned to the links for deeper more complex relations in the network, as well as parallel links or loops. Still, in practice it turns out that inspite the wild variety of 
networks, i.e. complex systems, most of them follow some universal way of organization [5].

It is observed that many real networks have small average path length, and small diameter compared to the number of nodes in the networks. In small world network most nodes are not neighbors of one another, but most nodes can be reached from any other node by a small number of steps. This phenomenon is discovered by Watts and Strogatz [3] at the end of the last century, and it is known as small world network.

Most biological and social networks show different properties. Namely, these networks have strong local clustering, or the neighbors of a given node are neighbors as well. High clustering coefficient suggests that nodes that have common neighbor are more likely to be neighbors than any other randomly chosen nodes. On the other side, humanmade networks like power grid, or networks repressenting some electronic circuits, have small clustering coefficient.

Another important network feature is the degree distribution. If the degree distribution obeys a power low, it is said that the network is scale-free. Another big class of networks have exponential degree distribution. Many networks were reported as scale free, but the evidence often remains inconclusive [6].

Among other common properties, it was shown [7] that real networks share another feature, self-similarity. This property is also observed in biological networks and some human made networks.

In order to study the properties of real networks, a numerous models have been proposed. Most of those models were stochastic [4], which requires probabilistic techniques to estimate the parameters of the studied network. However, a deterministic method turns to be useful, and its advantages are obvious; the parameters of the network can be express exactly and analytically. Dorogovtsev et al. [8] used mathematical objects and method to construct a deterministic network, and called it pseudo-fractal network. Their work was extended by Comellas and his colleagues $[9,10]$, and then by Zhang et al. [11, 12].

Here we give generalization of the model presented in [13]. In this article we present a deterministic method for construction of a class of self-similar networks. The elements of this class are obtained by vertex-transitive graphs, and an iterative construction procedure implemented on these graphs. This procedure is designed in a way that provides the property of self-similarity in the obtained network. Clearly, the properties of the obtained network will correlate with the properties of the starting graph.

\section{SELF-SIMILAR NETWORKS}

\section{A. Basic definitions}

In this section we first introduce the basic definitions in graph theory, and later we introduce some measures important for networks [14].

Let $G=(V, E)$ be a graph representing a network. The elements of $V$ are called vertices, and the elements of $E$ are edges of the graph $G$. Usually when networks are considered, vertices are called nodes and edges are called links. The order and size of a network (graph) are the cardinality of $V$ and $E$ respectively.

Two nodes $u$ and $v$ are adjacent if they share a common link (edge). The degree of a node $v$ is denoted by $\operatorname{deg}(v)$, and equals the number of nodes adjacent to $v$. The minimal degree in a graph $G$ is denoted by $\delta$, and the maximal degree by $\Delta$. If $\forall v \in V, \operatorname{deg}(v)=k, k \in N$, then the graph $G$ is called $k-$ regular. The distance between two nodes $u$ and $v$ in $G$, is the length of the shortest path between them. The distance between the nodes $u$ and $v$ in the graph $G$ is denoted by $\operatorname{dist}_{G}(u, v)$, or $\operatorname{dist}(u, v)$ if there is no place for confusion. The graph diameter is the maximal distance between any pair of nodes in a graph, i.e.

$$
\operatorname{diam}(G)=\max \{\operatorname{dist}(u, v) \mid u, v \in V(G)\} .
$$

Graph without loops and parallel links is called simple graph. A complete graph on $\boldsymbol{n}$ nodes, $\boldsymbol{K}_{\boldsymbol{n}}$, is a simple $\boldsymbol{n}-\mathbf{1}$-regular graph. A cycle on $\boldsymbol{n}$ nodes, $\boldsymbol{C}_{\boldsymbol{n}}$, is a simple 2-regular graph.

An automorphism, $f$, of graph $G$ is a bijection $f: V(G) \rightarrow V(G)$ such that any two vertices $u$ and $v$ of $G$ are adjacent if and only if $f(u)$ and $f(v)$ are adjacent. A graph $G$ is vertex-transitive [14], also sometimes called node symmetric [15], if for any two nodes $u, v \in V(G)$ there is an automorphism $f: V(G) \rightarrow V(G)$ such that $f(u)=v$. In other words, a graph is vertex-transitive if its automorphism group acts transitively upon its vertices. A graph is vertex-transitive if and only if its complement is vertex-transitive as well [16]. Cycles, complete graph, bipartite complete graphs $K_{n, n}$, Petersen graph etc. are all vertex-transitive graphs. 


\section{B. Clearly, all vertex-transitive are regular graphs, but not necessarily vice versa}

There are different measures that can be used for analyzing complex networks $[3,4,17]$. Here, we will focus on the degree distribution, clustering coefficient, and the diameter of the graph.

Let $\boldsymbol{P}(\boldsymbol{k})$ be the fraction of nodes of degree $\boldsymbol{k}$ in the network, and the order of the network. Simply $\boldsymbol{P}(\boldsymbol{k})$ can be interpreted as the probability that a node has degree $\boldsymbol{k}$. The degree distribution is the distribution of $\boldsymbol{P}(\boldsymbol{k})$ over all $\boldsymbol{k}$. The cumulative distribution, $\boldsymbol{P}_{\text {cum }}(\boldsymbol{k})$, is the number of nodes with degree at least $\boldsymbol{k}$ over the total number of nodes, i.e.,

$$
\boldsymbol{P}_{\text {cum }}(\boldsymbol{k})=\sum_{i=0}^{\boldsymbol{k}} \boldsymbol{P}(\boldsymbol{k}) .
$$

The clustering coefficient, $C(v)$, of a node $v$ of degree $\operatorname{deg}(v)$ in a graph (network) $G$ is defined in [10] as the number of triangles $\mathbf{e}_{v}$ with one node $v$ over the number of all pairs adjacent nodes to $v$, or

$$
C(v)=\frac{2 \mathbf{e}_{v}}{\operatorname{deg}(v)(\operatorname{deg}(v)-1)}
$$

The clustering coefficient is a measure of a degree to which nodes in a graph tend to cluster together [10].

\section{FRACTAL NETWORKS}

\section{A. Motivation - construction of fractal sets}

The aim of this paper is to construct a network with self-similar properties. In order to do so, we use similar technique as for construction of fractal sets. Iterated functional systems, or shortly IFS, are one possible method for fractal set construction [18]. For all terms not defined in this article consult Barnsley [18]. Formally iterated functional systems are finite sets of contractive mappings $f_{i}$ on complete metric space $X$, i.e.

$$
\left\{f_{i}: X \rightarrow X \mid i=1,2, \ldots, n\right\}, n \in N .
$$

The IFS defines Hutchinson operator as $H(S)=\bigcup_{i=1}^{n} f_{i}(S)$, where $S \subset X$ is non-empty compact set. Attractor of the iterated functional system is the unique nonempty compact set $A$ such that the following holds:

$$
A=\bigcup_{i=1}^{n} f_{i}(A) .
$$

Hence, the attractor $A$ is the fix set to the Hutchinson operator. Even more, for every nonempty compact set $S$ holds,

$$
A=\lim _{N \rightarrow \infty} H^{o N}(S),
$$

where

$$
\begin{gathered}
H^{o 0}(S)=S, \text { and } H^{o i}(S)=H\left(H^{o(i-1)}(S)\right), \\
i=1,2, \ldots, N .
\end{gathered}
$$

Figure 1 shows three iterations of an IFS with five contractions. The starting set is a square. The image in the right top corner is obtained after the first iteration, the image in the left bottom corner is obtained after two iterations. The attractor is shown in the right bottom corner.

The iterated functional systems allow fractals with condensation sets [18].
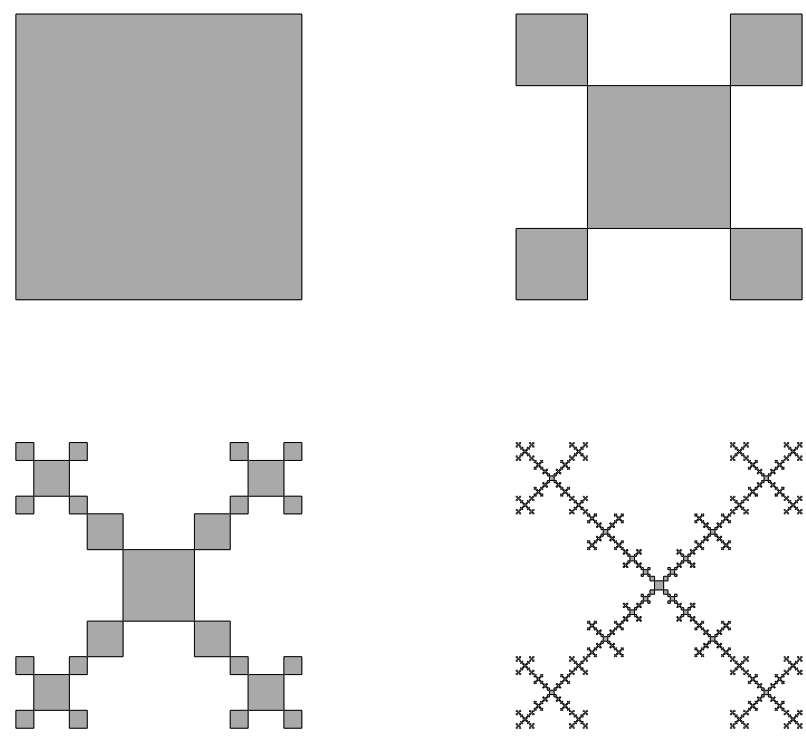

Fig. 1. The first three iterations of iterated functional system (with five mappings) applied on a square, and its attractor (bottom right)

\section{B. Network construction}

The construction method described here is based on vertex-transitive graphs. Let $G_{0}$ be the starting graph. In addition, assume that $G_{0}$ is vertextransitive graph on $n_{0}$ nodes, and $m_{0}$ edges. As noted in the introduction, this graph is a regular graph, so let the degree of each vertex of $G_{0}$ be denoted by $r$. Their relation is obtained by Hand-shake Lemma [14] and it reads as $2 m_{0}=n_{0} r$. We denote the diameter of $G_{0}$ by $d$. 
The networks that we are aiming to construct will be obtained in several iteration steps described below. Let $i$ and $j$ be non-negative integers $j \leq i$, and in the following text will refer to the iteration.

i. The graph $G_{1}$ is obtained when each node of $G_{0}$ is identified with a node of every new $a$ copies of $G_{0}, a \in N$.

ii, The graph $G_{j}$ is obtained when each node of $G_{j-1}$ is identified with a node of every new $a$ copies of $G_{0}$.

The network obtained in the $j$-th iteration, $G_{j}$, is constructed with consecutive applications of ii. Observe that due to vertex-transitivity of $G_{0}$ the obtained graphs $G_{j}, j \in N$, are unique up to isomorphism.

For simplicity $n_{j}, m_{j}$, and $d_{j}$ denote the number of nodes, edges and the diameter of $G_{j}$, respectively.

Figures 2-4 show a network obtained as describe above. The starting graph is a complete graph on 4 nodes, $G_{0}=K_{4}$, and $a=1$ (Figure 2). The graph obtained after one iteration is presented in Figure 3, and the one obtained after two iterations is shown in Figure 4.

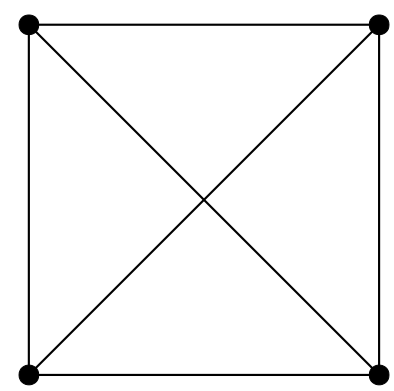

Fig. 2. The starting graph $K_{4}$, complete graph on 4 nodes

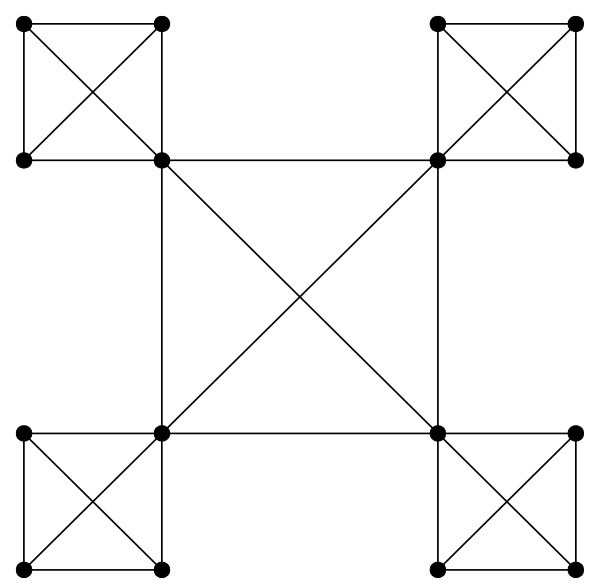

Fig 3. The graph obtained after the first iteration with starting graph $\boldsymbol{K}_{\mathbf{4}}$, and $\boldsymbol{a}=\mathbf{1}$

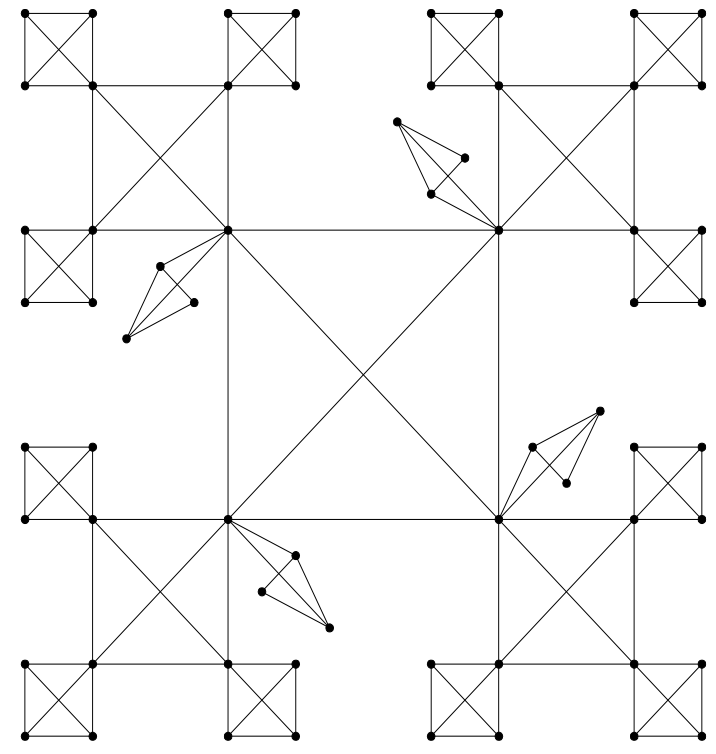

Fig. 4. The graph obtained after the first iteration, iteration with starting graph $K_{4}$, and $a=1$

\section{Networks propertis}

Our next interest is the number of nodes and edges in the network $G_{i}$, i.e. its size and order.

\section{Number of nodes (size)}

From the constructive rules described above, one obtains

$$
\begin{aligned}
\left|V\left(G_{i}\right)\right| & =\left|V\left(G_{i-1}\right)\right|+a\left|V\left(G_{i-1}\right)\right|\left(\left|V\left(G_{0}\right)\right|-1\right) \\
& =\left(1+\left(n_{0}-1\right) a\right)\left|V\left(G_{i-1}\right)\right| .
\end{aligned}
$$

Hence, we have the following recursion for the number of nodes

$$
n_{i}=\left(1+\left(n_{0}-1\right) a\right) n_{i-1}, \quad i \geq 1 .
$$

For simplicity let $p=1+\left(n_{0}-1\right) a$. Now, the solution of the last relation is

$$
n_{i}=p^{i} n_{0}, i \geq 1 \text {. }
$$

\section{Number of edges (order)}

Similarly like in the case for the number of nodes, we have

$$
\left|E\left(G_{i}\right)\right|=\left|E\left(G_{i-1}\right)\right|+a\left|V\left(G_{i-1}\right)\right|\left|E\left(G_{0}\right)\right| .
$$

Using (1) we get the recursive relation

$$
m_{i}=m_{i-1}+a m_{0} n_{0} p^{i} .
$$

Hence

$$
m_{i}=m_{0}\left(1+a n_{0} p+a n_{0} p^{2}+\cdots+a n_{0} p^{i}\right) .
$$




\section{Degree distribution}

Note that all nodes introduced in the same iteration have the same degree. Therefore it is important to consider numbers $\left|V\left(G_{j}\right)\right|-\left|V\left(G_{j-1}\right)\right|$ for $s$ positive integer. Namely, those numbers are precisely the number of nodes added in $j$-th iteration.

Let $G_{0}$ and $G_{i}, i>0$, be the starting and the final graph using the above construction, respecttively. Because of (1)

$$
\left|V\left(G_{j}\right)\right|-\left|V\left(G_{j-1}\right)\right|=n_{0} p^{j-1}(p-1), i \geq 1,
$$

and let denote the degree of all these nodes by $r_{i}$. Clearly,

$$
\operatorname{deg}(v)=r
$$

for a all nodes in the set $V\left(G_{i}\right) \backslash v\left(G_{i-1}\right)$,

$$
\operatorname{deg}(v)=r+a r
$$

for all nodes in the set $V\left(G_{i-1}\right) \backslash V\left(G_{i-2}\right)$, etc.

Observe that

$$
V\left(G_{i}\right)=\cup_{j=1}^{i} V\left(G_{j}\right) \backslash V\left(G_{j-1}\right) \cup V\left(G_{0}\right) .
$$

Hence, previous discussion implies the formula for the degree of the nodes: for

$$
\begin{gathered}
v \in V\left(G_{j}\right) \backslash V\left(G_{j-1}\right), 0 \leq j \leq i, \\
r_{j}=\operatorname{deg}(v)=r+(i-j) a r .
\end{gathered}
$$

In order to determine the cumulative degree distribution, $P_{\text {cum }}(k)$, we have to count the nodes with degree at least $k$ over the total number of nodes. A node will have a degree at the least $k$ if it is introduced in the $j$-th iteration, and

$$
j \leq i-\frac{k-r}{a r} .
$$

Now, according to [19], the cumulative degree distribution is the total number of nodes in $G_{j}$, where $j=i-\frac{k-r}{a r}$, divided with the number of noted in $G_{i}$. Hence we infer

$$
P_{\text {cum }}(k)=\frac{n_{j}}{n_{i}}=\frac{n_{0} p^{j}}{n_{0} p^{i}}=p^{j-i}=p^{-\frac{k-r}{a r}} .
$$

Equation (3) shows that the network constructed in this manner has exponential degree distribution.

\section{Average degree}

In most real networks there are small number of nodes that are connected with most of the nodes in the network, these are the nodes of high degree; and there is large number of nodes connected with very few nodes, probably with some of the nodes that are connected with most of the nodes. In the aspect of graph theory, most real networks have small number of nodes with high degree while there are large number of nodes with small degree. This characteristic of the real networks results with small average degree. Recall that in the considered network, the nodes introduced in the same iteration have same degree and the degree of the nodes introduced in $j$-th iteration is $r_{j}$. Clearly $r_{i}=r$, and

$$
r_{j}=r+(i-j) a r, \quad 0<i \leq j .
$$

Therefore the average degree of the network can be calculated as follows:

$$
\begin{aligned}
& \operatorname{deg}_{a v}\left(G_{i}\right)=\frac{1}{n_{i}} \sum_{j=0}^{i} n_{j}^{\prime} r_{j}= \\
& =\frac{1}{n_{i}}\left(n_{0}^{\prime} r_{0}+n_{1}^{\prime} r_{1}+\ldots+n_{i}^{\prime} r_{i}\right)=\frac{r}{n_{i}}\left(n_{0}(1+i a)+\right. \\
& \left.\quad+p a\left(n_{0}-1\right) \sum_{j=1}^{i} n_{0}^{j-1}(1+(i-j) a)\right)= \\
& =\frac{r}{p n_{0}^{i+1}+p n_{0}^{i}}\left(a p n_{0}^{i+1}+(1-a p) n_{0}^{i}+\right. \\
& \left.\quad+(1+i a) n_{0}^{2}-(a p+i+1+i a) n_{0}-1+a p\right) .
\end{aligned}
$$

From the last relation it is clear that the average degree tends to

$$
a\left(r-\frac{1}{n_{0}+1}\right)+\frac{1}{p\left(n_{0}+1\right)}
$$

as the number of iterations $i$ tends to infinity.

\section{Diameter}

Most of the real life network, share a common property, namely, most of these networks are "small world". For a network $G$ is said that it is a small world [3] if its diameter is at most $\log |V(G)|$.

Next, we determine the diameter of the constructed network. In order to determine the diameter of the network obtained after $i$ iterations, we use the property of self-similarity of the network. Recall that the diameter of the starting graph $G_{0}$ is $d$, and the fact that this graph is vertex-transitive. The 
graph obtained in after the first iteration, $G_{1}$ has diameter $3 d$. Due to the self-similarity property of the considered network we get that

$$
\operatorname{diam}\left(G_{i}\right)=\operatorname{diam}\left(G_{i-1}\right)+2 \operatorname{diam}\left(\mathrm{G}_{0}\right) \text {, for } i \geq 1 \text {. }
$$

By equation (4) we infer

$$
\operatorname{diam}\left(G_{i}\right)=(2 i+1) d, \quad i \geq 0 .
$$

The last equation suggests that the diameter of the obtained network grows linear with the number of iterations. The diameter of the network in terms of the number of vertices is given in the next relation

or

$$
\operatorname{diam}\left(G_{i}\right)=\left(2 \frac{\ln n_{i}-\ln p}{\ln n_{0}}+1\right) d,
$$

$$
\operatorname{diam}\left(G_{j}\right) \sim \ln n_{j} .
$$

Hence, this construction method results with a network that is small world.

\section{Clustering coefficient}

Our aim in this subsection is to determine the average clustering coefficient of the network. By definition

$$
C_{a v}\left(G_{i}\right)=\frac{1}{n_{i}} \sum_{v \in V\left(G_{i}\right)} C(v),
$$

where $C(v)$ denotes the clustering coefficient of the node $v$. We start with calculating the clustering coefficient of the node $v_{j}$ added in the $j$-th iteration.

Since the graph $G_{0}$ is vertex-transitive, all nodes $v$ have the same number of triangles (containing the node $v$ ), later on denoted with $t_{0}$. The same reasoning holds for all the nodes added in each iteration. Recall that the graph $G_{i}$ is constructed by identifying each node of $G_{i-1}$ with exactly one node of $a$ copies of $G_{0}$. This construction adds new triangles only among newly added nodes, to be more precise, only among nodes from the same copy of $G_{0}$, but does not create new triangles between nodes in $V\left(G_{j-1}\right)$ and $V\left(G_{j}\right) \backslash V\left(G_{j-1}\right)$. Now, let consider the number of triangles, $t_{j}$, containing a node $v$ (node added in $j$-th iteration) in the network $G_{i}, i \geq j$. The degree of $v$ is $r_{j}=r+(i-j) a r$, hence the node $v$ is a node in $1+(i-j) a$ subgraphs of $G_{i}$ isomorphic to $G_{0}$. Now, it is clear that the number of triangles with one vertex in $v$ equals

$$
t_{i}=(1+(i-j) a) t_{0} .
$$

This implies the clustering coefficient of the node $v$, i.e.,

$$
C(v)=\frac{2(1+(i-j) a) t_{0}}{r_{j}\left(r_{j}-1\right)}=\frac{2 t_{0}}{r(r+(i-j) a r-1)} .
$$

For the $i$-th iteration from $G_{0}$ one obtains a graph $G_{i}$, and the clustering coefficient of the network is as follows:

$$
C_{a v}\left(G_{i}\right)=\frac{1}{\left|V\left(G_{i}\right)\right|} \sum_{v \in V\left(G_{i}\right)} C(v) .
$$

For easier calculations we denote with $v_{j}$ a node added the $j$-th iteration. The number of such nodes is $n_{j}^{\prime}$. Now, the clustering coefficient of the network $G_{i}, C_{a v}\left(G_{i}\right)$ transforms to

$$
\begin{aligned}
& C_{a v}\left(G_{i}\right)=\frac{1}{n_{i}} \sum_{j=0}^{i} n_{j}^{\prime} C\left(v_{j}\right)= \\
& =\frac{2 t_{0}}{r p^{i}}\left(\frac{1}{r+i a r-1}+\sum_{j=1}^{i} \frac{(p-1) p^{j-1}}{r+(i-j) r a-1}\right) .
\end{aligned}
$$

For further calculations we introduce a new sequence

$$
g_{i}=\sum_{j=1}^{i} \frac{p^{j-1}}{r+(i-j) r a-1} .
$$

Our claim is that this is bounded and monotonic sequence of reals which would imply that the sequence $\left\{C_{a v}\left(G_{i}\right)\right\}_{i=1}^{\infty}$ is convergent. Trivial computation gives the relation

$$
g_{i+1}=\frac{1}{p^{i+1}} \frac{1}{r+i a r-1}+g_{i},
$$

which means that the sequence $\left\{g_{i}\right\}$ is monotonically increasing. Further more

$$
\frac{p^{i-1}}{p^{i}(r-1)} \leq g_{i} \leq \frac{1}{p^{i}} \sum_{j=1}^{i} \frac{p^{i-1}}{r-1}
$$

or

$$
\frac{1}{(r-1) p} \leq g_{i} \leq \frac{1}{(r-1)(p-1)}\left(1-\frac{1}{p^{i}}\right) \leq \frac{1}{(r-1)(p-1)},
$$

i.e. the sequence $\left\{g_{i}\right\}$ is bounded.

Finally

$$
\begin{gathered}
\frac{2 t_{0}}{r p^{i}} \frac{1}{r+i a r-1}+\frac{2 t_{0}(p-1)}{r(r-1) p} \leq C_{a v}\left(G_{i}\right) \leq \\
\leq \frac{2 t_{0}}{r p^{i}} \frac{1}{r+i a r-1}+\frac{2 t_{0}}{r(r-1)} .
\end{gathered}
$$

The sequence $\left\{C_{a v}\left(G_{i}\right)\right\}_{i=1}^{\infty}$ converges, and the limit, denoted with $C$, satisfies the estimate 


$$
\frac{t_{0}}{\left(\begin{array}{l}
r \\
2
\end{array}\right)} \frac{p-1}{p} \leq C \leq \frac{t_{0}}{\left(\begin{array}{l}
r \\
2
\end{array}\right)} .
$$

Observe that when $n_{0} \rightarrow \infty$ or $a \rightarrow \infty$, then $p \rightarrow \infty$ as well. Hence, we can conclude that graphs obtained with the procedure described in this article, from graph $G_{0}$ with large number of nodes or by adding large number of copies of $G_{0}$ in every iteration results with $C$ arbitrary close to $\frac{t_{0}}{\left(\begin{array}{l}r \\ 2\end{array}\right)}$.

\section{CONCLUSION REMARKS}

This paper is a continuation of the paper [13]. In order to give more freedom in the modelling of a network with wanted properties, here the starting graph is a vertex-transitive graph. At the same time we allow faster growth of the network, as $a \in N$ copies of the starting graph can be added to each node in every iteration. The properties of the resulting network, are closely correlated to the properties of the starting graph.

The obtained network is planar if and only if the starting graph $G_{0}$ is planar. Even more the network is outer planar if the same holds for $G_{0}$. The number of nodes, as well as the number of links grows exponentially in time, i.e. iteration (see equations (1) and (2)).

The network $G_{i}$ does not inherit the vertextransitivity, edge-connectivity and vertex-connectivity of $G_{0}$. The graph $G_{0}$ is vertex-transitive and $r$ regular graph, therefore the edge-connectivity of $G$ is equal to the degree $r$, while the vertex-connectivity will be at least $2(r+1) / 3$ [21]. Still, the network $G_{i}$ is not vertex-transitive, not even regular. The minimal degree of $G_{i}$ is $r$, while $\Delta=r+i a r$. The network $G_{i}$ has a vertex connectivity 1 , and edge connectivity $r$.

The constructed network has many features similar to real life networks. This network has exponential degree distribution (3). This property is based on the fact that the degree of each node grows linear in time. The exponential degree distribution is a common feature of many real life complex networks. The average degree of the network is

$$
a\left(r-\frac{1}{n_{0}+1}\right)+\frac{1}{p\left(n_{0}+1\right)}
$$

i.e. it depends on the starting graph and on the construction method. Still, as the number of iterations tends to infinity, the average degree of the networks is small. If the starting graph is big (has large number of vertices) or $a$ is big, then the average degree is approximately ar. In this network there are several nodes of high degree, incident with a lot of nodes of small degree. The nodes of small degree tend to be incident with high degree nodes. The considered network is a "small-world" as its diameter grows linear in time and it is a logarithmic function of the network size.

As far as the clustering is concerned, this construction gives a lot of freedom. The clustering coefficient of the network $G_{i}$ is approximately $\frac{t_{0}}{\left(\begin{array}{l}r \\ 2\end{array}\right)}$, where $\frac{n_{0} t_{0}}{3}$ is the number of triangles in the starting graph $G_{0}$, and $r$ is the degree of the nodes in $G_{0}$ (as it is regular). So, if the tendency is to construct a network with low clustering coefficient, one can chose a starting graph with small number of triangles (small $t_{0}$ ) compared with the degree of the nodes in the graph.

\section{REFERENCES}

[1] Saleh, M., Esa, Y., Mohamed, A.: Applications of complex network analysis in electric power systems, Energies, Vol. 11, 2018, DOI: 10.3390/en11061381.

[2] Saleh, M., Esa, Y., Onuorah, N., Mohamed, A. A.: Optimal microgrids placement in electric distribution systems using complex network framework, IEEE Conference Publication, 2017, pp. 1036-1040.

[3] Watts, D. J., Strogatz, S. H.: Collective dynamics of 'small-world' networks, Nature, Vol. 393, pp. 440-442 (1998).

[4] Barabasi, A.-L., Albert, R.: Emergence of scaling in random networks, Science, Vol. 286, pp. 509-512 (1999).

[5] Costa, L. da F. Rodrigues, F. A., Travieso, G., Boas, P. R. V.: Characterization of complex networks: A survey of measurements, Adv. Phys. Vol. 56, pp. 167-242 (2007).

[6] Clauset, A., Cosma, R. S., Newman, M. E. J.: Power-law distributions in empirical data, SIAM Review. Vol. 51, pp 661-703 (1997).

[7] Song, C., Havlin, S., Makse, H. A.: Self-similarity of complex networks, Nature, Vol. 433, pp. 392-395 (2005).

[8] Dorogovtsev, S. N., Goltsev, A. V., Mendes, J. F. F.: Pseudofractal scale-free web, Phys. Rev. E, Vol. 65, pp. 066122 (2002).

[9] Barriere, L., Comellas, F., Dalfo, C.: Fractality and the small-world effect in Sierpinski graphs, Journal of Physics, A: General Physics, Vol. 39 (38), pp. 117-139 (2006).

[10] Comellas, F., Miralles, A.: Modeling complex networks with self-similar outerplanar unclustered graphs, Physica A: Statistical Mechanics and its Applications, Vol. 388, pp. 2227-2233.

[11] Zhang, Z., Zhou, S., Chen, L.: Evolving pseudofractal networks, Eur. Phys. J. B, Vol. 58. pp 337-344 (2007).

[12] Zhongzhi, Z., Shuigeng, Z., Wenlei, X., Lichao, C., Yuan, L., Jihong, G.: Standard random walks and trapping on the 
Koch network with scale-free behavior and small-world effect, Physical Review E, Vol. 79, 061-113 (2009).

[13] Andova, V., Gegovska-Zajkova, S.: A deterministic construction of large networks using the Koch's snowflake. ETAI, 2013.

[14] Bondy, J. A.: U.S.R. Murty, Graph Theory, Springer, 2010.

[15] Chiang, W.-K., Chen, R.-J.: The $(n, k)$-star graph: A generalized star graph, Information Proc. Lett., Vol. 56, pp. 259-264 (1995)

[16] Bigss, N.: Algebraic Graph Theory, Cambrige University Press, 1993.
[17] Stanley, H. E., Amaral, L. A. N., Scala, A., Barthelemy, M.: Classes of small-world networks, PNAS. Vol. 97, 11149-52 (2000).

[18] Barnsley, M. F.: Fractals Everywhere, Academic Press, 1993.

[19] Newman, M. E. J.: The structure and function of complex networks, SIAM Review, Vol. 45, pp. 167-256 (2003).

[20] avasz, E., Barabasi, A. L.: Hierarchical organization in complex networks, Phys. Rev. E, Vol. 67, 026112 (2003).

[21] Godsil, C., Royal, G. F.: Algebraic Graph Theory, Springer, 2001. 